\title{
An exploratory, prospective cohort study of non-invasive dynamic quadripolar radiofrequency energy in vulvar lichen management. The new-generation $\mathrm{DQRF}{ }^{\mathrm{TM}}$ option
}

\author{
Vincenzo Prestia ${ }^{1}$, Elena Bertozzi ${ }^{2}$ and Mauro Raichi ${ }^{*}$ \\ ${ }^{1}$ ASST Melegnano Martesana, Gynaecology and Obstetrics, Italy \\ ${ }^{2}$ Pelvic Floor Physiotherapist, Sirio Clinic, Fidenza, Italy \\ ${ }^{3}$ Clinical Pharmacology and Biophysics Consultant, Milan, Italy
}

\begin{abstract}
Introduction: The main vulvar lichen treatment goal is itch reduction; improving and preserving the skin integrity and texture, healing the fissures and erosions, and preventing the later disfiguring fibrosis are the other crucial treatment goals. The side effect burden of high-potency topical corticosteroids, the current gold standard, can be heavy and alternative options are welcome. The paper illustrates the first exploratory outcomes in vulvar lichen planus and lichen sclerosus by the latest lowenergy technological evolution of radiofrequency treatments, Dynamic Quadripolar RadioFrequency ${ }^{\mathrm{TM}}$, in synergistic combination with in-depth penetration of active principles via Ultra-Pulsed Radioporation ${ }^{\mathrm{TM}}$.
\end{abstract}

Methods: Explorative cohort study in 58 ambulatory women 27 to 80 years old, eighteen nulliparous and 26 uniparous, $53.4 \%$ in the postmenopausal period. The $\mathrm{DQRF}{ }^{\mathrm{TM}}$ treatment program mainly aimed at symptom control, with four weekly sessions - 10 minutes over the lichenified vulvar areas and 10 minutes of vaginal rejuvenation even without evidence of lichen vaginal extension. Assessments (baseline and at the end of the treatment cycle): pain and dyspareunia (10-cm impromptu Visual Analogue Scales), vulvar burning (4-score Likert-like impromptu scale), vulvar lesions (binary evaluation - Yes / No).

Results: After a month, vulvar lichen inflammation appeared cooled down, and all cohort women reported dramatic improvements in daily and sexual symptoms variable between $-81.2 \%$ and $-92 \%$ for pain, burning and itching, and $-83 \%$ for dyspareunia.

Conclusions: Control of lichen inflammation, as documented by sequential photographs, is likely the rationale behind the very favourable outcomes after DQRF ${ }^{\mathrm{TM}}$ $+\mathrm{UPR}^{\mathrm{TM}}$ treatment. Well-designed comparative studies are critical to defining the role of the novel radiofrequency technology in vulvar lichen management.

\begin{abstract}
Abbreviations: IFN $\gamma$ : Interferon-gamma; CCL4, CCL5, CXCL9, CXCL10, CXCL11: Members of the 28-strong CC or $\beta$ class of chemokines; CXCR3, CCR5: Members of the ten known chemokine receptors (CCR1-10); CD4(+): T helper cells; CD8(+): Cytotoxic T cells, suppressor T cells; DQRF ${ }^{\mathrm{m}}$ : Dynamic QuadripolarRadioFrequency ${ }^{\mathrm{mn}}$; FOXP3: Forkhead box P3 (or scurfin, immune-tolerance related protein); IL-8: Interleukin-8; MDa: x106 dalton; mRNA: messenger ribonucleic acid; NF- $\kappa B$ : Nuclear Factor kappa-light-chain-enhancer of activated B cells; SEM: Standard error of the mean; TGF $\beta$ : Transforming Growth Factor beta; Th1: Type 1 T helper (Th1) cells; UPR ${ }^{\mathrm{m}}$ : Ultra-Pulsed Radioporation $^{\mathrm{m}}$; VAS: Visual Analogue Scales; VLP: Vulvar Lichen Planus; VLS: Vulvar Lichen Sclerosus
\end{abstract}

\section{Introduction}

Chronic ( $\geq 6$ weeks) skin pruritus troubles about $20 \%$ of Europeans; in $5 \%$ to $10 \%$ of them, the itching involves the vulva and female genitalia [1]. After candidiasis, the most common causes of vulvar pruritus are chronic dermatoses, including vulvar lichen sclerosus (VLS), vulvar lichen planus (VLP), and vulvar eczema [1]. VLS may account for up to one in 70 diagnoses by general gynaecology practitioners with diagnostic experience in VLP and VLS, yet the lag between symptoms and diagnosis, ranging from 5 to 15 years, suggests VLS is underdiagnosed or misdiagnosed for years $[2,3]$.
The link joining VLS and VLP is histopathology-the combination of a closely applied, band-like lymphocytic infiltrate and basal layer degeneration seen as apoptotic bodies, vacuolar change and squamatisation, which goes under the name of lichenoid reaction [4]. If the lichenoid reaction is associated with sawtooth-like rete ridges or acanthosis, the clinical variant is classic VLP; the clinical phenotype is erosive VLP if there is also a thinned or eroded epithelium. When the acanthosis is marked and associated with parakeratosis or hypergranulosis, the lichen variant is hypertrophic VLP. The evidence of multifocal or diffuse homogenised collagen in the papillary dermis signals VLS [4].

The well-demarcated, glazed erythematous lesions of erosive VLP, possibly with hyperkeratotic borders, usually distribute on the nonkeratinised squamous epithelium of labia minora and vestibule, but vaginal extensions and even vaginal scarring and adhesions are not unusual. The keratinised vulvar skin is the site of the spontaneously

${ }^{\star}$ Correspondence to: Mauro Raichi, MD, c/o Novavision Group SpA, Via dei Guasti, 29, 20826 Misinto, Italy, E-mail: mrdoc55@gmail.com

Key words: vulvar lichen sclerosus, vulvar lichen planus, Dynamic Quadripolar RadioFrequency, DQRF ${ }^{\mathrm{wn}}$, Ultra-Pulsed Radioporation ${ }^{\mathrm{Tw}}, U P R^{\mathrm{m}}$

Received: April 05, 2021; Accepted: April 20, 2021; Published: April 23, 2021 
Prestia V (2021) An exploratory, prospective cohort study of non-invasive dynamic quadripolar radiofrequency energy in vulvar lichen management. The newgeneration DQRF ${ }^{\mathrm{TM}}$ option

resolving, pruritic and multicolour papules and plaques of classic VLP and the thick violaceous plaques of hypertrophic VLP [4].

Conversely, the patchy, thin, glistening, ivory-white lesions, showing the Koebner phenomenon, of early VLS distribute over the labial, perineal, and perianal areas but spares all mucosal areas beyond the hymenal ring. Progressive pruritus is the leading symptom of a symptom cohort quite extended even in the early phases - vulvar burning, dyspareunia, apareunia, anorgasmia, dysuria, and genital bleeding [5]. The longer-term evolution is devastating: bleeding skin cracks and painful sore areas with secondary infections, scarring and narrowing of the vaginal introitus with flattening and loss of labia minora, burying of the clitoris up to clitoral phimosis and loss of all chances of gratifying intimacy, urinary retention with overactive bladder and stress urinary incontinence, and anal stenosis, obstruction, and constipation [5].

The first goal of therapy is reducing the itch; the second one is improving skin integrity and texture. Fissures and erosions must heal for the woman to resume everyday activities and enjoy once again a gratifying sexual life; unfortunately, the disappearance of all whitening is impossible and cannot be an explicit therapy goal. Preserving the vulvar architecture and preventing disfiguring changes are the third therapy goal [6]. Early and aggressive treatment with super-potent clobetasol propionate-like or potent mometasone furoate-like topical steroids, the usual therapeutic gold standards, may halt the progression and even induce regression, but local and systemic steroid side effects may be troublesome. Moreover, only about two-thirds of VLP and VLS patients comply with topical steroid therapy recommendations [7].

A new advanced radiofrequency-based vulvar remodelling strategy could overcome such side effect and compliance problems. The paper illustrates the first outcomes in a real-life exploratory pilot study in VLP/VLS patients with the latest technological evolution of radiofrequency treatments - Dynamic Quadripolar RadioFrequency $\left(D_{R R}{ }^{\mathrm{mix}}\right)$ combined with glucose gel/hyaluronic acid Ultra-Pulsed Radioporation $^{\mathrm{Ts}}\left(\mathrm{UPR}^{\mathrm{Ts}}\right)$.

\section{Methods}

\section{Real-life study design, exploratory rationale, and vulvar lichen cohort}

The study, aimed at symptom control and quality of life improvement in everyday life, including dyspareunia and overall discomfort during sexual activity, was carried out between November 2019 and November 2020 in an ambulatory setting, under the authors' responsibility and insurance coverage, in a prospective cohort of 58 successively enrolled women 27 to 80 years old. To improve the women's intimate life and control any vaginal lichen extension, the $\mathrm{DQRF}^{\mathrm{su}}$ treatment, using a coupling gel, also extended to the vaginal mucosa behind the hymenal ring, onto an area of about $20 \mathrm{~cm}^{2}$ as previously described $[8,9]$.

In the investigators' opinion, the study nature as the first exploratory assessment of the DQRF ${ }^{\mathrm{ru}}$ and $\mathrm{UPR}^{\mathrm{Tw}}$ technologies in treating vulvar lichen allowed for an uncontrolled prospective cohort design and simplified clinical scoring.

Inclusion criteria were a negative Papanicolau test and a VLP or VLP diagnosis certified by vulvoscopy and vulvar biopsy of sclerotic areas. Women candidate for enrolment should not have co-morbidities, including neoplasia, nor should have received conservative or invasive treatments including vulvectomy, cryosurgery and laser ablation. All enrolled women agreed, by signing an individual informed consent form, to the anonymous collection of their data and photographic evidence; they also agreed to the publication of study outcomes. All study materials were peer-reviewed for ethical problems, and the authors always safeguarded the full respect of the ethical standards laid down in the Declaration of Helsinki as revised in Brazil 2013.

\section{$\mathrm{DQRF}^{\mathrm{Tm}}$ and $\mathrm{UPR}^{\mathrm{Tm}}$ technologies and vulvar lichen}

The DQRF ${ }^{\mathrm{m} x}$-based EVA ${ }^{\mathrm{m} x}$ device (Novavision Group S.p.A., Misinto, Monza-Brianza, Italy), with its patented dynamic quadripolar technology and algorithmically controlled energy delivery to the gynaecological target areas, was previously described [8-10]. The four medically certified AISI 316 stainless steel dynamic quadripolar electrodes, mounted on the ergonomic probes of the DQRF ${ }^{\text {rx }}$ devices, steadily alternate between radio wave receiver and transmitter states with emitted frequencies in the range 1.0 to $1.3 \mathrm{MHz}$. The repelling electric fields so generated, when in the ideal configuration, concentrate the radiofrequency energy and the low-energy thermal effect in the subepithelial structures of the vulva and vaginal mucosa with high topographical and tridimensional precision. There is no need for a grounding pad because electric fields arise only within the closed and electronically controlled electrode system.

The energy emission settings were different to allow the control of local tissue temperatures in the vulvar lichen areas and the vaginal mucosa - between $8 \%$ and $10 \%$ of the device maximum, or no more than 5.5 watts out of a maximum of $55 \mathrm{~W}$, onto the vulvar target areas leading to subepithelial vulvar temperatures of 38 to $39^{\circ} \mathrm{C}$, and between $10 \%$ and $14 \%$ of the maximum, or no more than 7.7 watts, onto the vaginal mucosa leading to subepithelial vaginal temperatures of 39 to $41^{\circ} \mathrm{C}$. The back and forth and circular movements over the vestibule, labia minora, and the commissure were rapid to avoid pain; the total $\mathrm{DQRF}^{\mathrm{Tn}}$ energy delivery lasted 10 minutes onto the lichen vulvar areas, 10 minutes onto the vaginal mucosa.

Before the procedure, the only preparation was an alcohol-free cleanse with no analgesia or local anaesthesia (the DQRF $F^{\text {rix }}$ technology distinctly reduces Ohm's resistances in tissues). Figure 1 illustrates the EVA $^{\mathrm{m}}$ device and its vaginal and vulvar probes with DQRF ${ }^{\mathrm{m} w}$ electrodes. The procedure is performed with the woman on the examining table in the dorsal lithotomy position.

Patented movement and temperature sensors (RSS ${ }^{\mathrm{m}}$, Radiofrequency Safety System, proprietary technology) equip the

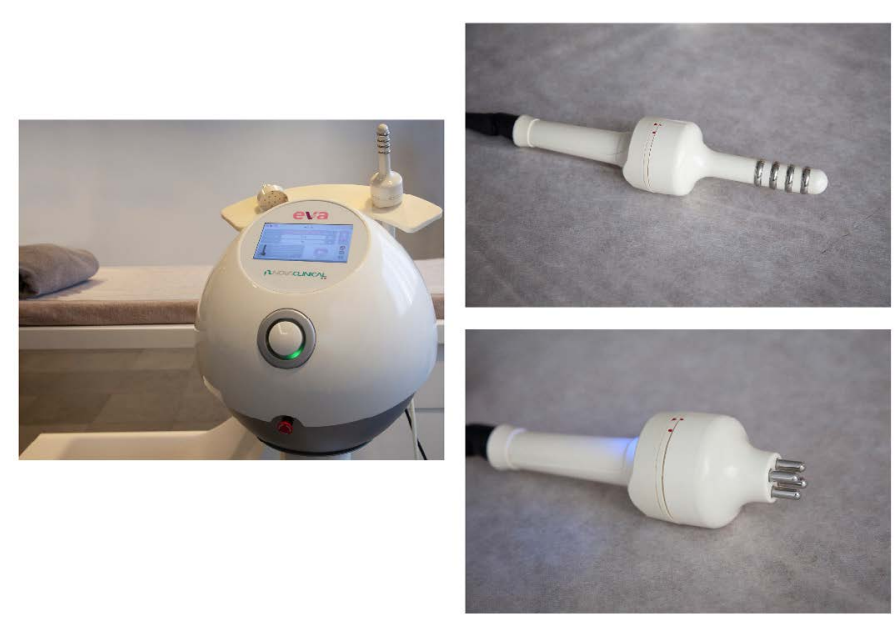

Figure 1. On the left: the EVA ${ }^{\mathrm{TM}}$ device with its double set of DQRFTM tips. Right upper photograph: evidence of the handpiece with the elongated, anatomical vaginal probe and its four stainless steel ring electrodes; right lower photograph: handpiece and vulvar probe; the electrodes are in a planar configuration 
Prestia V (2021) An exploratory, prospective cohort study of non-invasive dynamic quadripolar radiofrequency energy in vulvar lichen management. The newgeneration DQRF ${ }^{\mathrm{TM}}$ option

device to maximise safety. The device also exploits the proprietary $\mathrm{UPR}^{\mathrm{mx}}$ technology to deliver a lenitive and pro-trophic mixture of twothird glucose gel and one-third hyaluronic acid (molecular weight, 1.5 to $2.0 \mathrm{MDa}$, overall concentration $0.2 \%$ ), previously spread on the probe tip, to the target vulvovaginal areas [9].

\section{Timing of DRRF ${ }^{\mathrm{rm}}+\mathrm{UPR}^{\mathrm{Tw}}$ sessions and lichen assessments}

The cohort women underwent four weekly $\mathrm{DQRF}^{\mathrm{ma}}+\mathrm{UPR}^{\mathrm{m}}$ sessions, complemented by at-home self-administered perineal massage sessions.

Lichen assessments, performed before the first treatment session (baseline, T0) and at the end of the treatment cycle (T1), used 10-cm impromptu Visual Analogue Scales (VAS) for pain and dyspareunia, impromptu 4-score Likert-like scale for vulvar burning, and baseline and end-of-treatment photographs to highlight lichen lesions and their persistence.

A consolidation program (a monthly $\mathrm{DQRF}^{\mathrm{rax}}+\mathrm{UPR}^{\mathrm{rm}}$ session repeated for three months) and a long-term maintenance program (cycles of four DQRF ${ }^{\mathrm{sm}}+\mathrm{UPR}^{\mathrm{ma}}$ sessions repeated every six months) followed the end of the treatment cycle.

\section{Results}

On average, the 58 cohort women were $49.4 \pm 14.75$ years old (range $27-80$ years old, median $50 ; 31$ or $53.4 \%$ postmenopausal women), and the mean parity was 0.95 with 18 nulliparous, 26 uniparous, and 14 multiparous women. Forty-five women exhibited VLP or VLS lesions at baseline, with an overall heavy symptom burden-mean subjectively assessed pain score of $8.5 \pm 1.65$ out of 10 (median, 9), and burning and itching scores of, respectively, $2.5 \pm 0.63$ and $2.3 \pm 0.69$ out of 3 (medians, 3.0 and 2.0, respectively). The emotional impact on sexual life was also severe, with a mean baseline dyspareunia score of $8.8 \pm 1.55$ out of 10 (median, 9.5). Figure 2 illustrates the subjectively reported improvements of symptoms and dyspareunia after the DQRF ${ }^{\mathrm{m} x}+\mathrm{UPR}^{\mathrm{mm}}$ treatment cycle; Figure 3 some representative documentation of the VLP and VLS evolution between baseline and the end of the DQRF ${ }^{\mathrm{TN}}+$ $\mathrm{UPR}^{\mathrm{Ts}}$ treatment cycle.

When informally questioned during follow-up visits, most women reported a less troublesome daily life and gratifying subjective sensations of more hydration and more tissue elasticity already after the first $\mathrm{DQRF}^{\mathrm{m}}+\mathrm{UPR}^{\mathrm{m}}$ treatment session. Vulvar inflammation, visible in 45 cohort women out of 58 at baseline, had disappeared in all
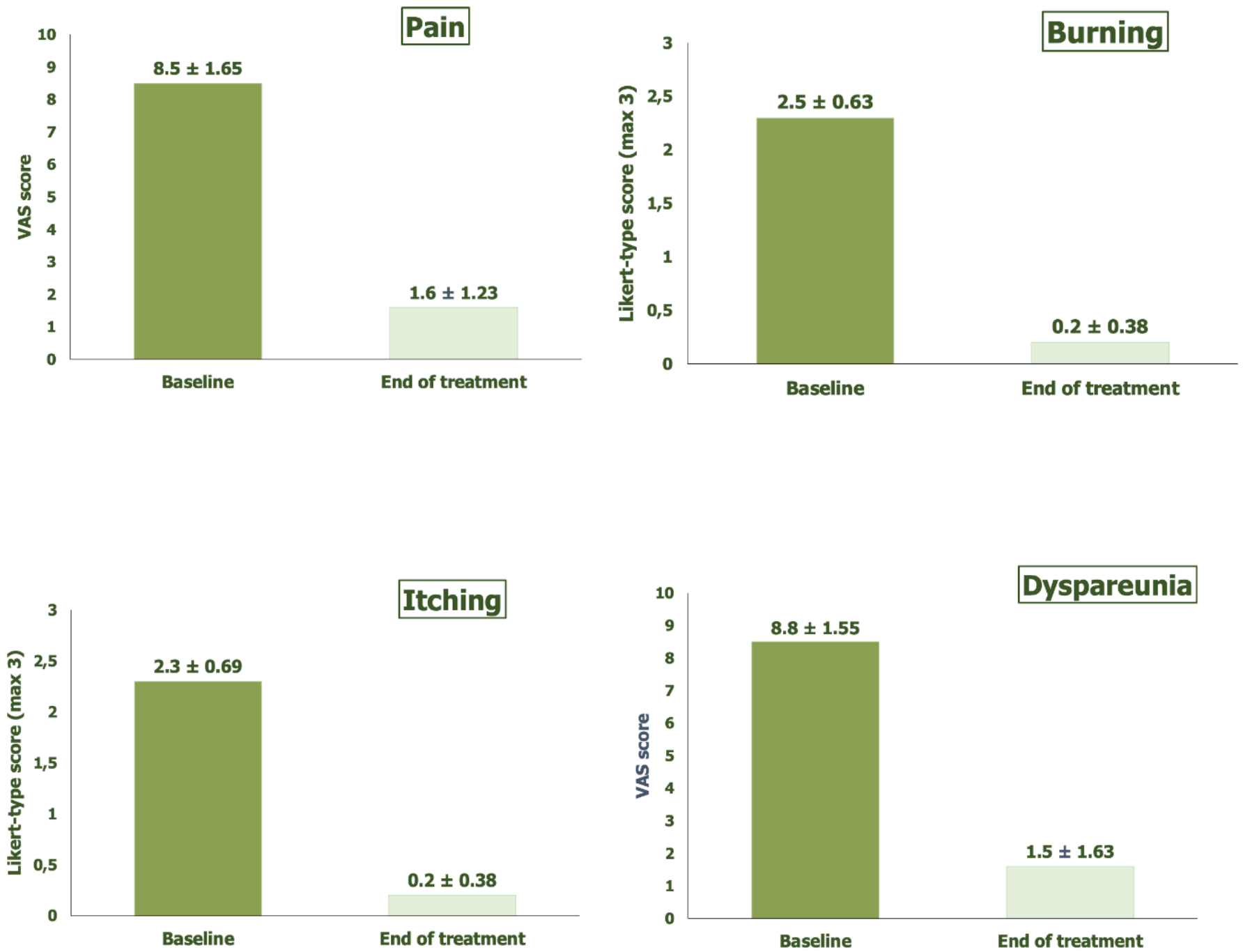

Figure 2. VLP and VLS symptom scores, end of treatment cycle vs baseline. Pain and dyspareunia: 10 -cm VAS, burning and itching: impromptu 0-3 scale; for all comparisons: $\mathrm{p}<0.01$ vs baseline) 
Prestia V (2021) An exploratory, prospective cohort study of non-invasive dynamic quadripolar radiofrequency energy in vulvar lichen management. The newgeneration DQRF ${ }^{\mathrm{TM}}$ option
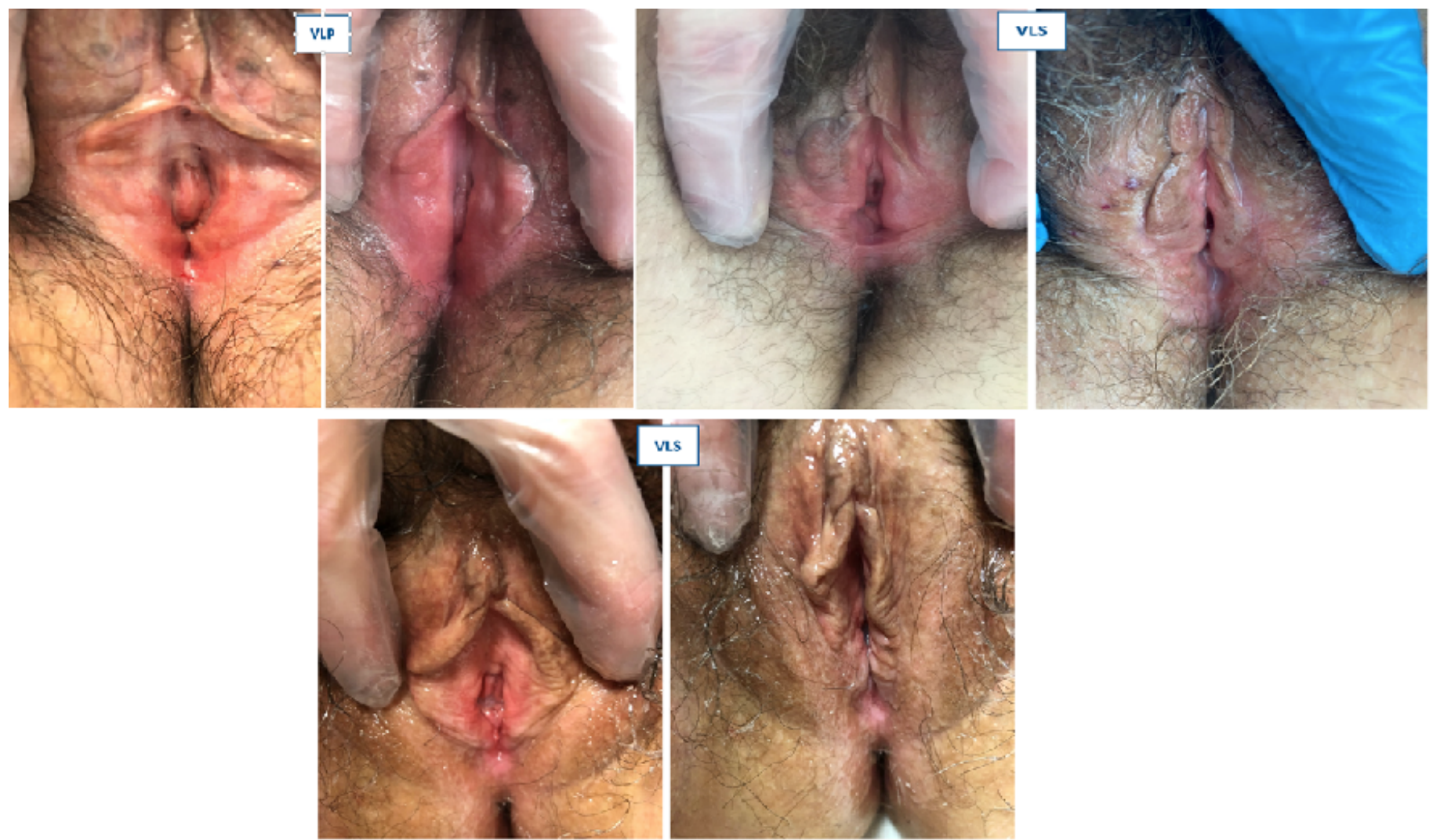

Figure 3. Representative examples of the vulvar morphology at baseline (photographs on the left) and the end of the DQRFTM + UPR ${ }^{\mathrm{TM}}$ treatment cycle (photographs on the right) with marked reduction of inflammation (VLS, vulvar lichen sclerosus; VLP, vulvar lichen planus)

women at the end of the treatment cycle. No woman reported burns, blisters or other fastidious side effects, neither during the ambulatory treatment sessions, always described as relaxing and comfortable, nor over the following days or weeks.

\section{Discussion}

Both VLP and VLS are T-cell-mediated chronic skin disorders and show a typical autoimmune phenotype, with a profile of increased proinflammatory cytokines - IFN $\gamma$, CXCR3, CXCL9, CXCL10, CXCL11, CCR5, CCL4, and CCL5 - signalling an IFN $\gamma$-induced Th1 immune response. Immunohistochemistry, showing a high density of CD4(+), CD8(+), and FOXP3(+) cells in the band-like lichenoid reaction in both conditions, confirms the strong T-lymphocyte response [11].

Symptom control and improvements in life quality rather than the impossible definitive cure are the treatment goals, exemplified by topical corticosteroids as still the therapeutic gold standard [6]. Many factors influence the compliance with topical steroid therapy-the greasy feeling and inaesthetic nature of ointments and creams, the trouble and confusions possibly associated with the variable dosing regimen over the early weeks and the shift to twice-weekly maintenance. Likewise, the ambiguous dosing instructions such as the vague "apply sparingly", the complicated application technique on medial labia majora, interlabial folds, both sides of labia minora and the perineum and not only "white" areas do not help compliance. Prompt relapses and flare-ups in most women, when overlooking the twice-per-week routine, are also liabilities of the gold standard topical steroid therapy [7]. All these are potent inducers to find alternatives to control VLP and VLS symptoms - effective and well-tolerated over an indefinite long term.
As known since 2004, thermal changes in collagen conformation and collagen denaturation induces neocollagenesis in the deep layers of the skin and subcutaneous tissues, signalled by the increased steady-state expression of collagen type I messenger RNA [12]. In the frequency range used in this exploratory study, radiofrequency may have other, possibly non-thermal effects of more prominence in lichen-like chronic inflammatory conditions-reduced keratinocyte proliferation and decreased expressions of TGF $\beta, N F-\kappa B$, IL- 8 , other pro-inflammatory cytokines and chemokines, and angiogenesisrelated inflammatory factors. These other effects are most likely the basis of the improvements observed after radiofrequency treatments in rosacea and acne lesions and scars $[13,14]$.

This study tentatively suggests the combined DQRF ${ }^{\mathrm{rx}}+\mathrm{UPR}^{\mathrm{in}}$ treatment strategy might indeed be a safe and effective alternative to the corticosteroid gold standard in vulvar lichen. The side effect and discomfort profiles were nil; the same is true for the women's compliance to complete the treatment cycle with no recorded drop-off.

In terms of efficacy, a few $\mathrm{DQRF}^{\mathrm{m}}+\mathrm{UPR}^{\mathrm{m} \omega}$ sessions cooled down lichen inflammation in all women, as reported by the investigators and documented by sequential photographs; concomitantly, all cohort women scored dramatic improvements in their heavy baseline symptom burden and sexual lives-possibly too dramatic. Together with the open, uncontrolled design and the short follow-up, assessing relief from lichen symptoms only through impromptu, non-validated VAS and Likert-like scales is a critical study weak point with the need for a strong caveat against too hasty conclusions. 
Prestia V (2021) An exploratory, prospective cohort study of non-invasive dynamic quadripolar radiofrequency energy in vulvar lichen management. The newgeneration DQRF ${ }^{\mathrm{TM}}$ option

Lichen symptoms severely disrupt the daily life and self-image of affected women. However dramatic were improvements over the few study weeks, all reported symptom reliefs were subjective and thus liable to an uncontrolled placebo effect-the main reason why the study can in no way claim to establish a new vulvar lichen management standard. As an exploratory study, it has the only ambition to suggest a rationale for the $\mathrm{DQRF}^{\mathrm{m}}+\mathrm{UPR}^{\mathrm{m}}$ treatment option. The contributing role of in-depth radioporation of the lenitive and possibly pro-trophic glucose gel and hyaluronic acid is also unclear, whilst there seem to be few doubts about the value of at-home perineal massage sessions as frequently as possible to maintain tissue elasticity.

Well-designed studies must confirm this exploratory study's subjective outcomes over the long term; correctly designed "dosefinding" studies are also critical to define the ideal $\mathrm{DQRF}^{\mathrm{max}}+\mathrm{UPR}^{\mathrm{m}}$ schedule and how frequently repeat the treatment cycles-yearly? More frequently? Still a question mark. Future studies will also have to explore the biophysics of the DQRF ${ }^{\mathrm{ma}}$ anti-inflammatory effect, which presumably is the basis of the rapid and dramatic relief from daily and sexual lichen symptoms enthusiastically hailed by the DQRF ${ }^{\mathrm{Tm}}$ treated women.

\section{Conclusions}

Control of lichen inflammation is likely the rationale behind the favourable outcomes after $\mathrm{DQRF}^{\mathrm{mat}}+\mathrm{UPR}^{\mathrm{mix}}$ treatment. Well-designed studies are warranted to define the role of the new non-invasive radiofrequency technology in vulvar lichen management.

\section{Acknowledgements}

Novavision Group SpA, Italy, is the worldwide patent holder of the

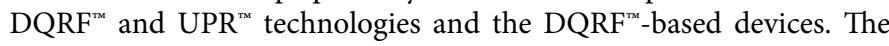
authors wish to acknowledge Novavision Group SpA for their support with the publication costs.

\section{Publishable conflict of interest statement}

The authors declare that they have no competing or conflicts of interest relating to what described in the paper.

\section{Disclosure of source(s) of financial support}

No direct financial support. Novavision Group SpA, Via dei Guasti, 29, 20826 Misinto, Italy, the worldwide patent holder of the Dynamic Quadripolar radiofrequency $\left(\mathrm{DQRF}^{\mathrm{rx}}\right)$ and Ultra-Pulsed Radioporation $\left(\mathrm{UPR}^{\mathrm{Tm}}\right)$ technologies and the $\mathrm{DQRF}^{\mathrm{mm}}$-based devices will fully support publications costs by Obstetrics and Gynecology Reports if the manuscript is accepted - the only financial support the authors will receive.

Institutional Review board status - Not relevant. The manuscript reports on the real-life prospective treatment of unselected ambulatory subjects (no formal inclusion or exclusion criteria) freely seeking radiofrequency relief from vulvar lichen symptoms and attendant disruption of everyday quality of life. The Clinical Report Form and Informed Consent Forms were discussed and reviewed by peers at the National Health Service territorial facility, where the first author, Dr Vincenzo Prestia, works. The authors performed the study in complete agreement with the Declaration of Helsinki. No unusual risk was reasonably foreseeable in participating subjects other than those incurred with the usual ambulatory radiofrequency procedures routinely performed for vulvar lichen management. In any case, the authors' professional malpractice insurance coverage during private practice activities lawfully protected the subjects from all risks.

\section{Authors' contribution statement}

All authors sought and got informed consents from the women subjects seeking radiofrequency treatment because of vulvar lichen problems and enrolled in the study. All women received informed consents about the benefits and risks they could reasonably expect from the $\mathrm{DQRF}^{\text {rax }}$ procedure. All authors performed all vulvar lichen procedures. All authors are accountable for the clinical and editorial work's accuracy and integrity, leading to the manuscript's submission to Obstetrics and Gynaecology Reports, including all comments on outcomes.

\section{References}

1. Woelber L, Prieske K, Mendling W, Schmalfeldt B, Tietz H-J, et al. (2020) Vulvar pruritus - causes, diagnosis and therapeutic approach. Dtsch Arztebl Int 116: 126-133. [Crossref]

2. Lewis FM, Tatnall FM, Velangi SS, Bunker CB, Kumar A, et al. (2018) British Association of Dermatologists guidelines for the management of lichen sclerosus, 2018. Br J Dermatol 178: 839-853. [Crossref]

3. Krapf JM, Mitchell L, Holton MA, Goldstein AT (2020) Vulvar lichen sclerosus: current perspectives. Int $J$ Womens Health 12: 11-20. [Crossref]

4. Day T, Wilkinson E, Rowan D, Scurry J, ISSVD Difficult Pathologic Diagnoses Committee (2020) Clinicopathologic diagnostic criteria for vulvar lichen planus. J Low Genit Tract Dis 24: 317-329. [Crossref]

5. N Singh, P Ghatage (2020) Etiology, Clinical features, and diagnosis of vulvar lichen sclerosus: a scoping review. Obstet Gynecol Int 2020: 7480754. [Crossref]

6. American College of Obstetricians and Gynecologists' Committee on Practice Bulletins -Gynecology (2020) Diagnosis and management of vulvar skin disorders: ACOG Practice Bulletin, number 224. Obstet Gynecol 136: e1-e14. [Crossref]

7. Lee A, Bradford J, Fischer G (2015) Long-term management of adult vulvar lichen sclerosus: a prospective cohort study of 507 women. JAMA Dermatol 151: 1061-1067. [Crossref]

8. Vicariotto F, Raichi M (2016) Technological evolution in the radiofrequency treatment of vaginal laxity and menopausal vulvovaginal atrophy and other genitourinary symptoms: first experiences with a novel dynamic quadripolar device. Minerva Ginecol 68: 225-236. [Crossref]

9. Tranchini R, Raichi M (2018) Ultra-Pulsed Radioporation further enhances the efficacy of Dynamic Quadripolar RadioFrequency in women with postmenopausal vulvovaginal atrophy. Clin Obstet Gynecol Reprod Med 4: 1-5.

10. Vicariotto F, DE Seta F, Faoro V, Raichi M(2017) Dynamic quadripolar radiofrequency treatment of vaginal laxity/menopausal vulvovaginal atrophy: 12-month efficacy and safety. Minerva Ginecol 69: 342-349. [Crossref]

11. Terlou A, Santegoets LAM, van der Meijden WI, Heijmans-Antonissen C, Swagemakers SMA, et al. (2012) An autoimmune phenotype in vulvar lichen sclerosus and lichen planus: a Th1 response and high levels of microRNA-155. J Invest Dermatol 132: 658-666. [Crossref]

12. Zelickson BD, Kist D, Bernstein E, Brown DB, Ksenzenko S, et al. (2004) Histological and ultrastructural evaluation of the effects of a radiofrequency-based nonablative dermal remodeling device: a pilot study. Arch Dermatol 140: 204-209. [Crossref]

13. Son M, Park J, Oh S, Choi J, Shim M, et al. Radiofrequency irradiation attenuates angiogenesis and inflammation in UVB-induced rosacea in mouse skin. Exp Dermatol 14115. [Crossref]

14. Min S, Park SY, Yoon JY, Suh DH (2015) Comparison of fractional microneedling radiofrequency and bipolar radiofrequency on acne and acne scar and investigation of mechanism: comparative randomised controlled clinical trial. Arch Dermatol Res 307: 897-904. [Crossref]

Copyright: (C2021 Prestia V. This is an open-access article distributed under the terms of the Creative Commons Attribution License, which permits unrestricted use, distribution, and reproduction in any medium, provided the original author and source are credited. 